of the study, study participants will be offered the opportunity to participate in 2-3 hour focus groups to discuss acceptability of the product as a treatment for premalignant HPV-related cervical disease until data saturation is achieved. Power and sample size calculations are based on the primary outcome of interest, which is clearance of HPV at 6 months. Basu et al (2013) documented HPV clearance in as many as $80 \%$ of subjects with topical curcumin. To account for an expected lower success rate in HIV-infected women, who will also be included in the study, we intend to power our study to determine a more conservative $20 \%$ improvement in clearance rate at 6 months with curcumin treatment, assuming an expected clearance rate of HPV in HIV-infected women of $25 \%$. In order to detect a $20 \%$ difference of HPV clearance among those treated with intravaginal curcumin vs. placebo at 6 months, about 80 patients per arm would achieve $80 \%$ power at the $5 \%$ significance level. To account for up to $20 \%$ loss to follow-up or discontinuation, the total sample size in each arm would be 100 subjects with a total of approximately 200 subjects enrolled in both arms. RESULTS/ANTICIPATED RESULTS: We are currently in the process of collecting data for this study. We hypothesize that intravaginal curcumin will have a $20 \%$ higher rate of HPV clearance at 6 months as compared to placebo. Primary outcome measures will include clearance of HPV at 6 months in curcumin vs. placebo. Secondary outcomes measures will include recurrence of disease by either cytologic or histologic abnormality requiring further surveillance or treatment at 6 months. We also hypothesize that intravaginal curcumin administered once weekly at bedtime for 20 weeks will be safe, acceptable, and well tolerated. This is based off of previous findings from the Phase 1 trial of intravaginal curcumin that we performed. During this Phase 1 trial, we explored daily intravaginal administration of $2000 \mathrm{mg}$ of curcumin to further understand curcumin's tolerability. Our focus group participants displayed an overwhelming consensus that daily administration affected quality of life, specifically due to the yellow-colored vaginal discharge from the medication. Study participants expressed that once or twice weekly administration was more tolerable and feasible. Our proposed study would therefore test the tolerability and effects of weekly curcumin administration and its ability to clear HPV infection. The primary outcome measure will be the proportion of study participants who discontinue treatment for any reason (acceptability) and the proportion of study participants who discontinue treatment due to adverse effects (tolerability). DISCUSSION/SIGNIFICANCE OF IMPACT: Non-surgical treatments that decrease the morbidity and risk of progression of premalignant HPV-related cervical disease are greatly needed, especially in low-resource settings and among women experiencing barriers to care and/or at high risk for disease progression. Medical treatment with the natural herb curcumin is an emerging strategy that may allow subjects to receive treatment of cervical lesions without undergoing a surgical procedure.Several preclinical and clinical studies have shown curcumin's ability to reduce tumors and precancerous lesions in animal and human cancer cells. Curcumin can suppress the activation of transcription factor NF- $\mathrm{KB}$ and the expression and activity of VEGF and p16INK4a, biomarkers known to be elevated in premalignant HPV-related cervical disease.Studies have also shown that curcumin alters HPV-associated molecular pathways in cancer cells, suppressing cervical cancer growth by inhibiting the transcription of oncoproteins HPV16 and HPV18 (designated as E6 and E7) and restoring $\mathrm{p} 53$ and retinoblastoma function. Our proposed study would therefore test the tolerability and effects of weekly curcumin administration and its ability to clear HPV infection. Our results will generate novel data as to what is an acceptable and well-tolerated dosing regimen of intravaginal curcumin, which would be crucial in designing further curcumin intervention studies. The results of our proposal will explore the effect of intravaginal curcumin as a standalone and adjuvant therapy to a LEEP among women with premalignant HPV-related cervical disease. The potential to not just excise diseased tissue, but to directly augment the clearance of the causative agent HPV, would have profound long-term ramifications in resource-limited settings and among women experiencing barriers to care and/or at high risk for disease progression.

4185

\section{Assessing Barriers to Retention in Care Continuum Among HCV Positive Homeless Adults of New Orleans \\ Riley Eli Santiago ${ }^{1}$, and Janna Wisniewski ${ }^{2}$ \\ ${ }^{1}$ Tulane University School of Medicine- LA CaTS; ${ }^{2}$ Tulane University}

OBJECTIVES/GOALS: This study has two primary aims: 1) evaluate points of success and failure in connecting hepatitis $\mathrm{C}$ virus (HCV) positive homeless patients to care following a preliminary positive rapid $\mathrm{HCV}$ test result, and 2) describe the barriers cited by patients who drop out at each step in the care continuum. METHODS/STUDY POPULATION: A retrospective longitudinal analysis of adult (18 years or older) homeless individuals accessing shelter at six homeless shelters in New Orleans, LA was conducted. Every patient who came through a testing site received a survey collecting information on demographics, barriers to healthcare, and recent utilization of health services. A retrospective chart review of hospital and homeless clinic medical records was used to track patient linkage to care and their progress through the HCV care continuum. We defined successful linkage to care as attendance at the first scheduled follow-up appointment for treatment with a primary care physician. RESULTS/ANTICIPATED RESULTS: A total of 1719 unique patients were identified from August 2016 through August 2019 which included 36\% self-identified as African American/Black, 55\% identified as White and $8 \%$ identified as mixed-race or other. A total of $24 \%$ of individuals reported no insurance coverage while $66 \%$ of patients reported having insurance. Overall, 85 patients reported they experienced no barriers to healthcare. Of those who reported barriers, $44 \%$ reported trouble with finances or insurance, $22 \%$ transportation, $18 \%$ personal drug use, $9 \%$ personal alcohol use, and $7 \%$ reported a distrust of healthcare providers or the system. Other barriers included long wait times, distance, and recent incarceration. DISCUSSION/SIGNIFICANCE OF IMPACT: Although screening for $\mathrm{HCV}$ is readily available, barriers exist which prevent diagnosis and treatment. We implemented a HCV testing and linkage-to-care program between local homeless shelters and health centers in New Orleans in an effort to reduce HCV-related morbidity and mortality.

Assessment of ivermectin-treated backyard chickens as a novel urban West Nile virus prevention strategy

Karen Holcomb ${ }^{1}$, Chilinh Nguyen ${ }^{2}$, Brian D. Foy ${ }^{2}$, and Christopher M. Barker $^{1}$

${ }^{1}$ University of California, Davis; ${ }^{2}$ Colorado State University

OBJECTIVES/GOALS: We conducted a randomized field trail to evaluate the efficacy and safety of a novel vector control strategy that 